\title{
The Flow Field Analysis and Flow Calculation of Ultrasonic Flowmeter Based on the Fluent Software
}

\author{
Ling Guo, ${ }^{1,2}$ Yue Sun, ${ }^{1}$ Ling Liu, ${ }^{3}$ Zhixi Shen, ${ }^{1}$ Ruizhen Gao, ${ }^{1}$ and Kai Zhao \\ ${ }^{1}$ School of Automation, Chongqing University, Chongqing 400044, China \\ ${ }^{2}$ Logistic Engineering University, Chongqing 400016, China \\ ${ }^{3}$ Chongqing Vocational Institute of Engineering, Chongqing 400037, China \\ Correspondence should be addressed to Zhixi Shen; shenzhixi@cqu.edu.cn
}

Received 26 January 2014; Accepted 3 March 2014; Published 22 May 2014

Academic Editor: Xiaojie Su

Copyright (c) 2014 Ling Guo et al. This is an open access article distributed under the Creative Commons Attribution License, which permits unrestricted use, distribution, and reproduction in any medium, provided the original work is properly cited.

\begin{abstract}
We can build the three-dimensional structure model based on the Gambit software and achieve the distribution of flow field in the pipe and reflux flow condition at the position of transducer in regard to the real position of transducer according to the Fluent software. Under the framework, define the reflux length based on the distance of reflux along the channel and evaluate the effect of reflux on flow field. Then we can correct the power factor with the transmission speed difference method in the ideal condition and obtain the matching expression of power correction factor according to the practice model. In the end, analyze the simulation experience and produce the sample table based on the proposed model. The comparative analysis of test results and simulation results demonstrates the validity and feasibility of the proposed simulation method. The research in this paper will lay a foundation for further study on the optimization of ultrasonic flowmeter, enhance the measurement precision, and extend the application of engineering.
\end{abstract}

\section{Introduction}

Compared with the conventional flowmeter, the ultrasonic flowmeter has a better performance since it has no moving parts, no pressure loss, wide measuring range, excellent repeatability, and high precision [1], and it is widely used in industrial production $[2,3]$, especially for large diameter pipes and larger flows $[4,5]$. The ultrasonic flowmeter is mainly comprised of an ultrasonic transducer installed on the measuring pipe and the related sensors of temperature and pressure [6]. The ultrasonic transducer has two installations: intrusive and nonintrusive $[7,8]$. With the nonintrusive installation, the signal emitted by the ultrasonic transducer needs to go through the pipe wall twice, which will weaken the strength of the signal largely, while the low SNR will affect the stability and accuracy of signal receiving. The intrusive installation is currently used in normal situations [9]. For the single-path ultrasonic flowmeter, the intrusive installation requires a through-hole in the pipe wall, where the ultrasonic transducer can be built. This structure and ultrasonic transducer generate disturbance in the flow field, cause measuring errors, and may be the key problem in the measurement of ultrasonic flowmeter. Reference [10] pointed out that the unevenness near the pipe wall induced by the ultrasonic transducer distorts the flow field and leads to lower measuring values. The measuring value would be lower by $0.05 \%$ while the length of the channel is $5 \mathrm{~m}$; the measuring value would be lower by $0.35 \%$ while the length of the channel is $1 \mathrm{~m}$. However, for the pipe with small diameter and low flow, the length of channel will be shorter, far less than $1 \mathrm{~m}$; the reference had not stated the magnitude of error. Raišutis [11] analysed the flow at the recess in the pipe with a diameter of $70 \mathrm{~mm}$; the flow field was distorted and the symmetry of the velocity distribution was destroyed; this also influenced the measurement of flow. Yet the velocity of flow was large in this reference, and the Reynolds number was large; this belonged to the turbulent flow. Zhang et al. [6] and Zheng et al. [12] did research on the non-flow-calibrated method of ultrasonic flowmeter, using the computational fluid dynamics numerical simulation method, and analyzed the influences of DN500-the multichannel transducer ultrasonic flowmeter-on the accuracy of measurement. The analysis 


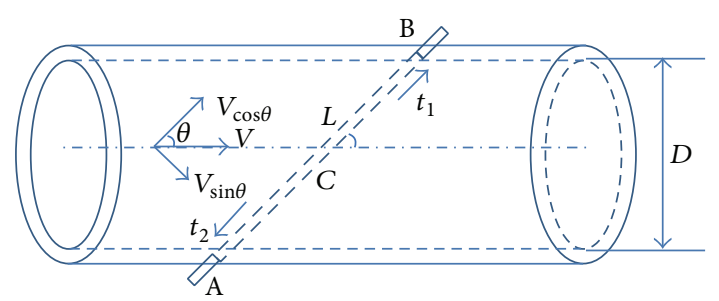

FIgURE 1: The diagram of the principle of ultrasonic flowmeter.

of flow field pointed out that since there might be a reflux near the transducer, the average measurement of velocity of each channel was lower, and the measuring values of flows were lower as well.

With analysis of the related references, we found that (1) the ultrasonic flowmeter uses double-path and multipath measurement generally $[13,14]$; the shortcomings can be listed as follows: on one hand the complex pipe structure requires higher accuracy of installation; on the other hand the use of multiple ultrasonic transducers will increase the costs; (2) few researches have been done for the fluid with low Reynolds number in the single-path ultrasonic flowmeter. And for this kind of ultrasonic flowmeter, the intrusive installation and transducer have nonignorable disturbance on flow field.

In order to estimate the measuring errors caused by disturbance, this paper proposed a novelty model that builds the practice structure of a single-path ultrasonic transducer with a $50 \mathrm{~mm}$ pipe diameter and calculates the disturbance of transducer to the flow field approximately using the Fluent software for flow field analysis combined with test data; based on the above model we can analyze the measurement effects on the accuracy by quantitative methods.

This paper is structured in the following way. In Section 2, the measurement principle of the single-path ultrasonic flowmeter is presented. In Section 3, we can model and analyze the flow field based on Fluent software. The simulation results demonstrate the effectiveness and generality of the proposed algorithm in Section 4. Finally, Section 5 summarizes the conclusion.

\section{Measurement Principle of the Single-Path Ultrasonic Flowmeter}

2.1. Operational Principle. We can see the measurement principle of transmission speed difference method in the single-path ultrasonic flowmeter [15-20] from Figure 1. The diameter of the pipe is represented by $D$, ultrasonic transducers are installed on A and B sides, which could emit and receive the ultrasonic signals, $L$ represents the distance of A and $\mathrm{B}$, and $\theta$ is the angle of $\mathrm{AB}$ with the pipe axis. It will need time $t_{1}$ for the signal from $A$ to $B$ and the circuit delay is $\tau_{1}$. For the same reason, the signal will cost time $t_{2}$ from B to A and the circuit delay is $\tau_{2}$; in addition, the actual pressure is $P$ and the actual temperature is $T$.

It is assumed that the fluid will flow with velocity $V$ and the direction is parallel to the axis to the right, so on the channel $L$ the propagation velocity of the ultrasonic signal is composited by the acoustic velocity $C$ and component of flow velocity $V_{\cos \theta}$, then the propagation time of ultrasonic signal in both downstream and upstream directions can be shown, respectively:

$$
\begin{gathered}
\text { Downstream: } t_{1}=\frac{L}{C+V_{\cos \theta}}, \\
\text { Upstream: } t_{2}=\frac{L}{C-V_{\cos \theta}} .
\end{gathered}
$$
by

Using (1), the linear mean velocity $V_{L}$ will be calculated

$$
V_{L}=\frac{L}{2 \cos \theta}\left(\frac{1}{t_{1}-\tau_{1}}-\frac{1}{t_{2}-\tau_{2}}\right)
$$

Because of the presence of the actual fluid velocity distribution in the pipe cross-section, linear mean velocity $V_{L}$ is not equal to the cross-section mean velocity $V_{\mathrm{A}}$. Assume that there is a power correction factor $K$ between the linear mean velocity $V_{L}$ and the cross-section mean velocity $V_{\mathrm{A}}$, the expression is that

$$
K=\frac{V_{\mathrm{A}}}{V_{L}}
$$

Then we can get that the flow of the pipe is

$$
Q=K \frac{\pi D^{2}}{4} V_{L}
$$

Considering the influences of pressure and temperature, the flow can be converted under the standard working conditions:

$$
Q=K \frac{\pi D^{2}}{4} V_{L} \cdot \frac{P}{P_{0}} \cdot \frac{T_{0}}{T}
$$

2.2. Model of Ideal Channel. Based on the hydrodynamic theory, the fluid has viscosity so that the fluid shows different velocities at the points of different diameter in the crosssection. And the Reynolds number can be the only parameter that distinguishes moving patterns of viscous fluid. Whether the fluid moving as laminar or turbulent flow can be decided by the value of Reynolds number, there is a lower bound around 2000 for the critical Reynolds number, which transits laminar flow to turbulence. In the moving of laminar flows, the tiny disturbance in the flow field such as the roughness of pipe wall and free changes of surface will attenuate gradually so that the fluid flows as laminar flow. However, the tiny disturbance can be increased and flow becomes unstable if Reynolds number is bigger, so it is difficult to make sure the final status after disturbance increased as the equations are of nonlinearity, we can only conclude that the final stage is connected with structure of flow field and Reynolds number.

With regard to the ideal laminar flow shown in Figure 1, the fluid may flow symmetrically if the gravity effects are ignored, and the velocity will be a function of radius $r$ in 


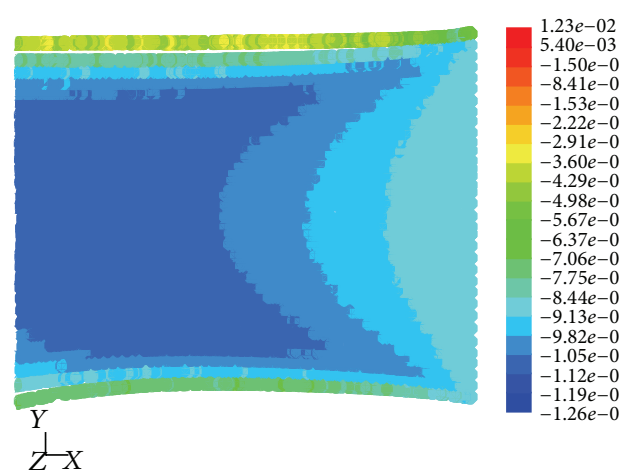

FIGURE 2: The velocity profile in the laminar flow.

the horizontal direction. Presume that the pressure drop on the pipe is $\Delta P$ and the radius of the pipe $R=D / 2$, the velocity distribution at cross-section can be shown by the HagenPoiseuille formula:

$$
u=\frac{\Delta P}{4 \mu \mathrm{L}}\left(R^{2}-r^{2}\right) .
$$

Based on the equation above, each point velocity distributed parabolically with radius $r$; the largest velocity is on the pipe axis as $r=0$ :

$$
u_{\max }=\frac{\Delta P}{4 \mu \mathrm{L}} R^{2}=\frac{\Delta P}{16 \mu \mathrm{L}} D^{2} .
$$

Through the simulation, we can get the flow results with parabolic distribution in Figure 2; the distribution is shown clearly.

According to the distribution of flow velocity, the crosssectional area of the flow can be calculated as

$$
d Q=u d \mathrm{~A}=\frac{\Delta P}{4 \mu \mathrm{L}}\left(R^{2}-r^{2}\right) 2 \pi r d r .
$$

After integration:

$$
Q=\int_{0}^{R} \frac{\Delta P}{4 \mu \mathrm{L}}\left(R^{2}-r^{2}\right) 2 \pi r d r=\frac{\pi \Delta P}{128 \mu \mathrm{L}} D^{4} .
$$

The mean flow velocity at cross-section can be presented as

$$
V_{\mathrm{A}}=\frac{\mathrm{Q}}{\mathrm{A}}=\frac{\Delta P}{32 \mu \mathrm{L}} D^{2}=\frac{1}{2} u_{\max } .
$$

Under the normal circumstances, the path of ultrasonic flowmeter is installed in the middle of the pipe, then the linear mean velocity is

$$
V_{L}=\frac{1}{L} \int_{L} u(r) d L=\frac{1}{R} \int_{0}^{R} u_{\max }\left(1-\frac{r^{2}}{R^{2}}\right) d r=\frac{2}{3} u_{\max } .
$$

On the basis of (4), (10), and (11), we can compute the power correction factor $K$ :

$$
K=\frac{V_{\mathrm{A}}}{V_{L}}=\frac{3}{4} .
$$

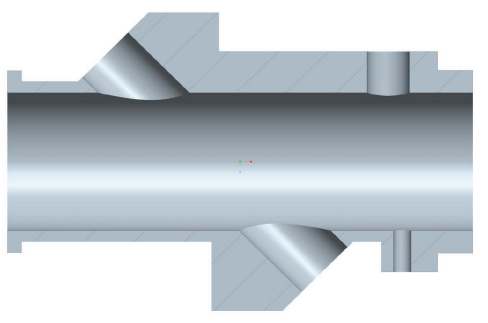

FIgURE 3: The cross-section of ultrasonic flowmeter.

We can achieve the relationship between the cross-section mean velocity, linear mean velocity, and the maximum flow rate based on the above theory; meanwhile the relationship between the cross-section mean velocity and linear mean velocity is obtained. However, the magnitude and position of maximum velocity cannot be measured directly in practice and engineering application.

\section{Fluent-Based Modeling and Analysis of Flow Field}

The laminar flow velocity distribution and the value of power correction factor have been derived under the ideal circumstances. However, the pipe is not smooth in practice, and the pipe will be installed with temperature and pressure sensors inside it, which may disturb the flow field making the velocity of flow field dissatisfy the standard parabolic distribution. Therefore, the power correction factor $K$ is not a fixed value.

In this paper, we design the actual structure of ultrasonic flowmeter with small diameter and small flow as shown in Figure 3. Then, the model processing of the simulation and modeling is as follows.

In the first step, we can use the Gambit software to build the geometric model of the flowmeter. The pipe is cylindrical with a $50 \mathrm{~mm}$-diameter with holes at the 45-degree angle along with pipe axis, where the transducer is installed; the pressure and temperature sensors are built separately inside the two holes on the left side.

Secondly, mesh the model. Since the pipe has a throughhole structure that the transducer and sensors are installed in, the shape of flow field is not cylindrical any more. Thence, the surface and volume of flow field can ensure the grid near the transducer and sensors is dense enough and can control the number of grids by choosing tetrahedral mesh.

Next, put the grid file into the Fluent software in order to do the fluid calculation. As the pipe is of small diameter, small flow, and small Reynolds number, we should employ the laminar flow model to make the fluid calculation.

At last, set the parameters for calculations. Using the Fluent software to deal with the laminar flow model when the minimum flow is $0.6 \mathrm{~m}^{3} / \mathrm{h}$ and the corresponding Reynolds number $R_{e}$ is 140 . Based on the calculation above, we can set 


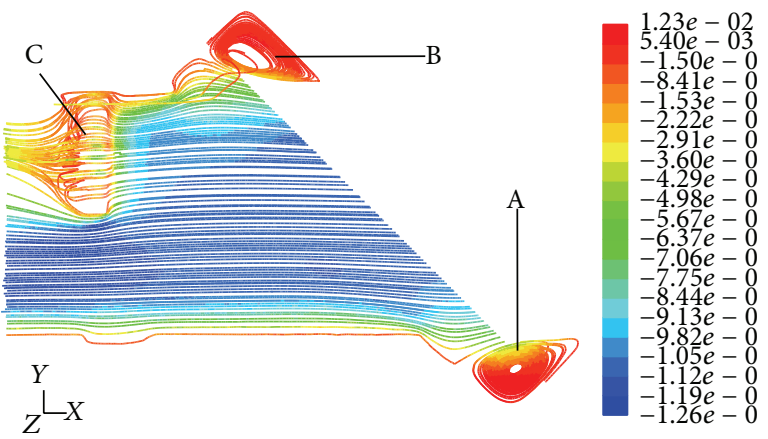

FIGURE 4: The diagram of sound channel $\left(R_{e}=145\right)$.

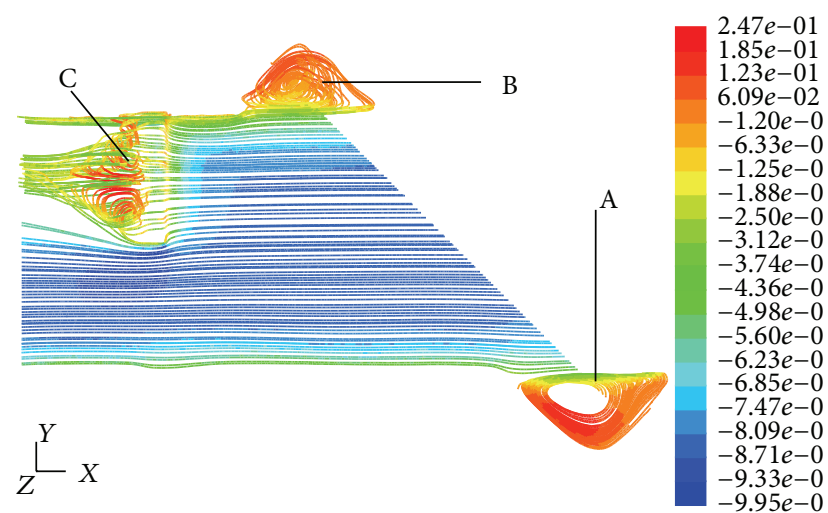

FIGURE 5: The diagram of sound channel $\left(R_{e}=1168\right)$.

the uniform speed entrance and free exit to do the simulated calculation and analyze the output after convergence.

3.1. The Disturbance of Pipe Structure in the Flow Field. In Figures 4 and 5, the fluid flows into the pipe from the right side and the flow field will be affected by the structures of the transducer and the sensor installed therefore generating reflux near the attachments of transducer and sensor at point $\mathrm{A}$ and $\mathrm{B}$. The strength of reflux is changing every time according to Reynolds number and it will be increased when Reynolds number is bigger. The reflux will go through the test channel, produce opposite flow, and decrease the linear average velocity of the path, which may affect the measuring accuracy directly. For flowmeter with large diameter, the influences of reflux can be ignored generally. But these influences may be significant with the small-diameter and small-flow condition.

In addition, the fluid velocity has parabolic distribution in the ideal laminar flow model and is parallel to the axis, but in the actual structure we can get the curve of fluid velocity along $A B$ in Figures 6 and 7. In Figure 6, velocity is not symmetrically distributed in the $X$ direction along $A B$. At point $\mathrm{A}$ the velocity is exactly positive which means the fluid flows to the opposite direction. The reflux will have larger influence at point $A$ than point B. Figure 7 shows that particles were distributed along the velocity to the $Y$ axis

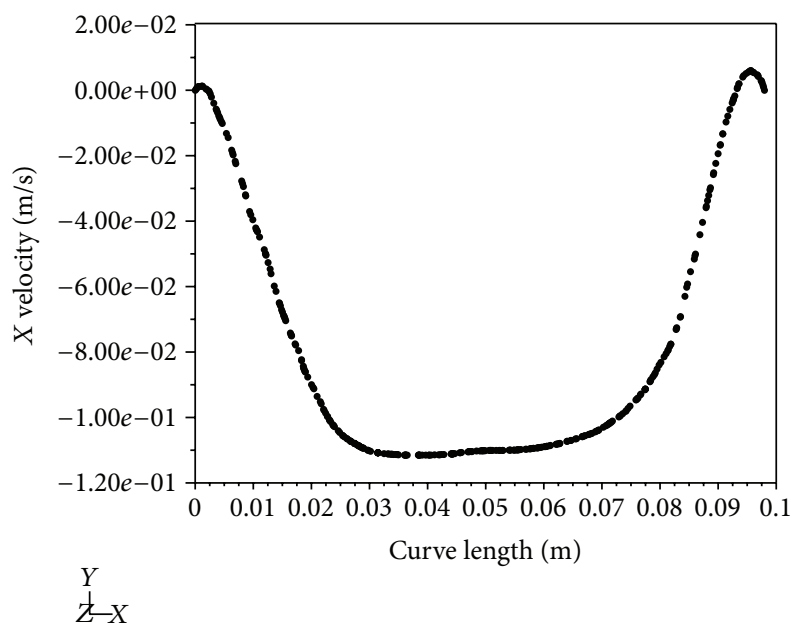

Figure 6: The velocity curve in the $X$ direction along AB.

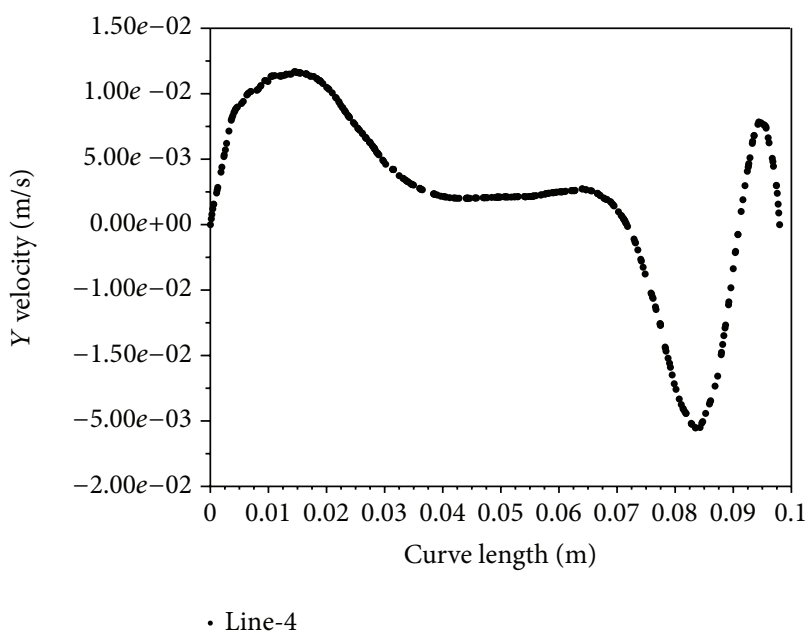

FIgURE 7: The velocity curve in the $Y$ direction along AB.

along $\mathrm{AB}$; on the axis of pipe there is tiny flow to the $Y$ axis, but apparent flow to the $Y$ axis exists near the ends of $\mathrm{AB}$.

The curve of cross-sectional velocity distribution at the midpoint of output pipe (the midpoint of $\mathrm{AB}$ ) can be seen in Figure 8. Being influenced by actual pipe structure and transducer, it is no more standard parabolic distribution.

3.2. Estimating the Influence of Reynolds Number on Reflux. To the fluid, the Reynolds number can be estimated by

$$
R_{e}=\frac{\rho V R}{\eta},
$$

where $R_{e}$ is the Reynolds number, $\rho$ is the density of gas, $V$ is velocity of flow, and $R$ means the radius of the pipe.

In the model shown in Figure 3, the distance $A B$ for transducer installation is $0.098 \mathrm{~m}$, the air viscosity is $1.84 e-$ $05 \mathrm{~Pa} \cdot \mathrm{S}$, and the density can be seen as $1.225 \mathrm{~kg} / \mathrm{m}^{3}$. According to whether the particles on the line in the trajectories of $\mathrm{AB}$ are circulated or not, we can ensure the length of reflux on 


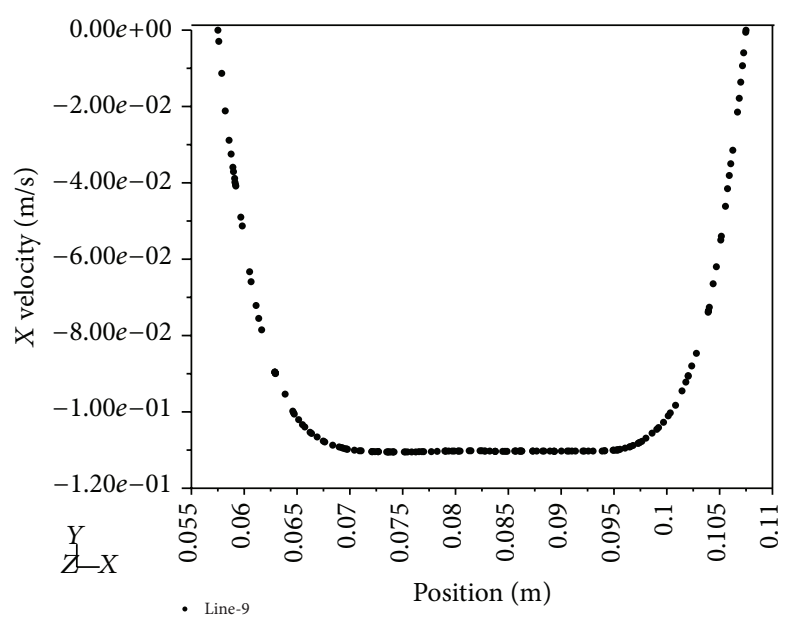

FIGURE 8: The curve of cross-sectional velocity distribution at the midpoint of output pipe $A B$.

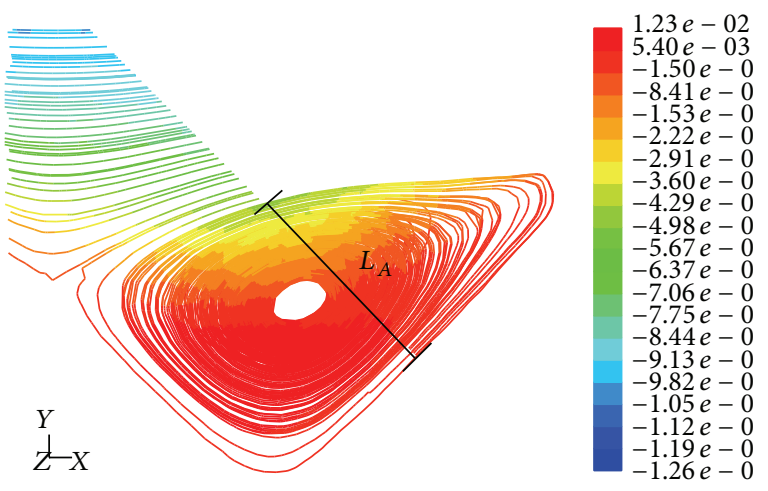

Figure 9: The length of reflux at point A.

TABLE 1: The length of refluxes $L_{\mathrm{A}}$ and $L_{\mathrm{B}}$.

\begin{tabular}{lcccc}
\hline Number & $R_{e}$ & $L_{\mathrm{A}}$ & $L_{\mathrm{B}}$ & Percentage (\%) \\
\hline 1 & 145 & 0.01191 & 0.00798 & 20.3 \\
2 & 226 & 0.01198 & 0.00815 & 20.5 \\
3 & 459 & 0.01212 & 0.00956 & 22.1 \\
4 & 1168 & 0.01339 & 0.01110 & 25.0 \\
5 & 1853 & 0.01451 & 0.01361 & 28.7 \\
\hline
\end{tabular}

the propagation path. At the same time, the length of reflux at point $\mathrm{A}$ is $L_{\mathrm{A}}$ and at point $\mathrm{B}$ is $L_{\mathrm{B}}$, as in Figures 9 and 10.

Simulating under different Reynolds numbers, we can get the reflux of fluid at the transducer and the length of refluxes $L_{\mathrm{A}}$ and $L_{\mathrm{B}}$; the statistics are expressed in Table 1.

On the basis of Table 1, the length of reflux will be raised if Reynolds number is larger. The curve that shows the relationship between Reynolds number and length of reflux is drawn in Figure 11.

3.3. Power Correction Factor Analysis. From (12) above, the power correction factor $K$ plays an important role in measurement accuracy of ultrasonic flowmeter, which is the key parameter of ultrasonic flowmeter calibration [9]. The

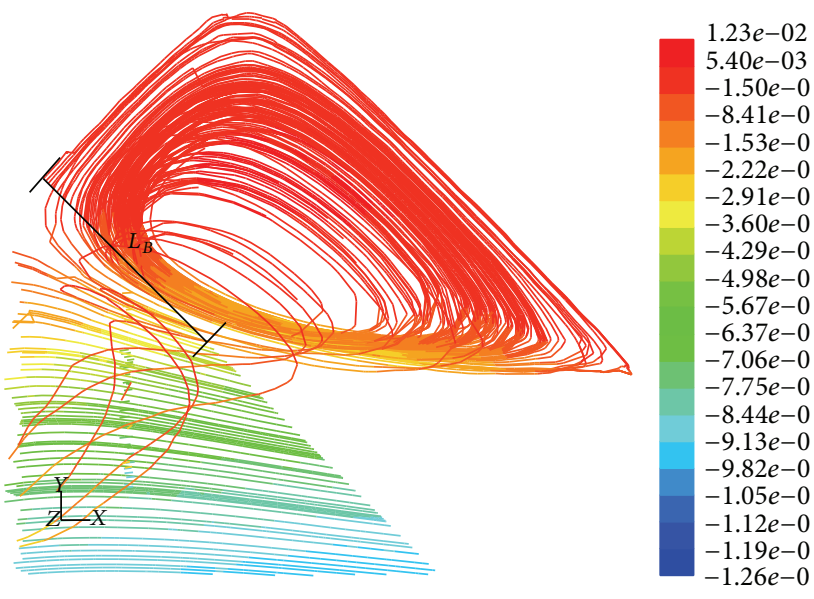

FIgURE 10: The length of reflux at point B.

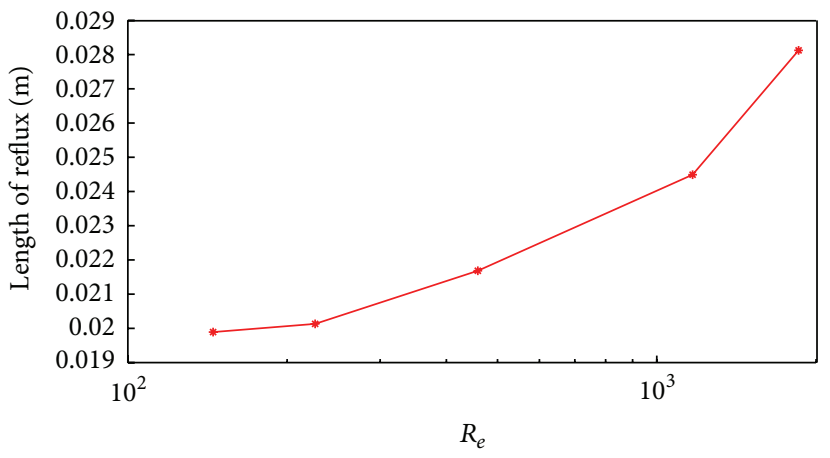

FIGURE 11: The relationship diagram of $R_{e}$ and length of reflux.

value of $K$ is highly related to the Reynolds number, pipe structure, and other factors. If the pipe structure is certain, $K$ changes all the time when the Reynolds number changes. And among the references related to the power correction factor $K$, two assumptions can be concluded.

First, assume that the fluid is flowing parallel to the pipe axis in Figure 1. But in practice, the fluid direction is influenced by the pipe shape; it will not certainly and completely be parallel to axis; the velocity $V$ of transducer and sensor is not in the horizontal direction. If the actual flowing direction is not parallel to the axis, according to (5), the measurement will generate large errors.

Second, suppose that the pipes are all smooth tubes; we can ignore the influences on the fluid of exact pipe structure. However, because of the actual structure of the transducer by intrusive installation, especially for the pipes with small diameters, the fluid flow will be affected.

In engineering, we can get the power correction factor generally from the test when correcting the flowmeter against the fluid with low Reynolds number, if, considering the actual shape, structure of pipe, and the influences on the measurement of the non-axis-parallel flowing fluid, the relationship between flow field that affects power correction factor and measurement error of pipe flow can be analyzed. 
Reflux makes the linear average velocity less so that the measurement is lower and the error is negative. Now considering the influences of reflux, we can rewrite (2):

$$
\begin{aligned}
V & =\frac{L-L_{\mathrm{A}}-L_{\mathrm{B}}}{2 \cos \theta}\left(\frac{1}{t_{1}-t_{\mathrm{A} 1}-t_{\mathrm{B} 1}-\tau_{1}}-\frac{1}{t_{2}-t_{\mathrm{A} 2}-t_{\mathrm{B} 2}-\tau_{2}}\right) \\
& =\frac{L-L_{\mathrm{A}}-L_{\mathrm{B}}}{2 \cos \theta} \frac{t_{2}-t_{\mathrm{A} 2}-t_{\mathrm{B} 2}-\tau_{2}-t_{1}+t_{\mathrm{A} 1}+t_{\mathrm{B} 1}+\tau_{1}}{\left(t_{1}-t_{\mathrm{A} 1}-t_{\mathrm{B} 1}-\tau_{1}\right)\left(t_{2}-t_{\mathrm{A} 2}-t_{\mathrm{B} 2}-\tau_{2}\right)} \\
& =\frac{L-L_{\mathrm{A}}-L_{\mathrm{B}}}{2 \cos \theta} \frac{\Delta T_{\mathrm{sim}}-\Delta T_{\mathrm{A}}-\Delta T_{\mathrm{B}}+\left(\tau_{1}-\tau_{2}\right)}{\left(t_{1}-t_{\mathrm{A} 1}-t_{\mathrm{B} 1}-\tau_{1}\right)\left(t_{2}-t_{\mathrm{A} 2}-t_{\mathrm{B} 2}-\tau_{2}\right)} .
\end{aligned}
$$

Considering that the type and size of the transducer in part $\mathrm{A}$ are generally the same as part $\mathrm{B}$, so the hardware delay can be regarded as the same: $\tau_{1}=\tau_{2}$. Then

$$
V=\frac{L-L_{\mathrm{A}}-L_{\mathrm{B}}}{2 \cos \theta} \frac{\Delta T_{\mathrm{sim}}-\Delta T_{\mathrm{A}}-\Delta T_{\mathrm{B}}}{\left(t_{1}-t_{\mathrm{A} 1}-t_{\mathrm{B} 1}-\tau_{1}\right)\left(t_{2}-t_{\mathrm{A} 2}-t_{\mathrm{B} 2}-\tau_{2}\right)} .
$$

According to the simulation output data we can get $t_{1}, t_{\mathrm{A} 1}, t_{\mathrm{B} 1}, t_{2}, t_{\mathrm{A} 2}$, and $t_{\mathrm{B} 2}$. Since $\tau_{1}$ and $\tau_{2}$ are errors caused by circuit board delay, which can be ignored, getting the linear average velocity by calculation, then the power correction factor $K$ is calculated basing on (3) and (4).

\section{Simulation}

To test the effectiveness of simulation analysis, make a trial version of ultrasonic flowmeter shown in Figure 3; then test with the nozzle flow calibration test device.

4.1. Time Difference Correction of Ultrasonic Propagation. The analysis from the last section leads to the conclusion that the actual structure of the pipe generates reflux at points $A$ and $\mathrm{B}$; the reflux raises the downstream ultrasonic propagation time and lowers the upstream time so that the time difference is less, the flow measurement is lower, the errors will be negative, with the same diameters, and the measuring errors will increase gradually along with the increasing entrance velocity.

To estimate the exact influences on measurement of reflux, this paper is based on the output of Fluent and counts the propagation time and time difference of ultrasonic wave between two transducers, as Table 2 states. From the table, the time difference of reflux at point $\mathrm{A}$ is $\Delta T_{\mathrm{A}}$, the time difference of reflux at point $B$ is $\Delta T_{\mathrm{B}}$, the downstream and upstream time difference through the $\mathrm{AB}$ channel is $\Delta T_{\text {sim }}$, and the unit is nanosecond (ns).

4.2. Power Correction Factor K. The power correction factor $K$ is calculated based on (3) and (4), and the results are shown in Table 3.

Data from Table 3 suggests that the power factor will change in the same direction with Reynolds number. This also proves that power factor $K$ may have negative errors using ideal model and the errors increase as Reynolds number
TABLE 2: Time difference of ultrasonic wave at points $\mathrm{A}$ and $\mathrm{B}$.

\begin{tabular}{lccccc}
\hline Number & $R_{e}$ & $\Delta T_{\mathrm{A}}$ & $\Delta T_{\mathrm{B}}$ & $\Delta T_{\text {sim }}$ & Percentage (\%) \\
\hline 1 & 145 & 1.874 & 1.608 & 97.5 & 3.6 \\
2 & 226 & 3.283 & 1.883 & 147.2 & 3.5 \\
3 & 459 & 5.60 & 3.810 & 277.1 & 3.4 \\
4 & 1168 & 17.723 & 2.366 & 683.4 & 2.94 \\
5 & 1853 & 23.10 & 1.59 & 1037.8 & 2.38 \\
\hline
\end{tabular}

TABLE 3: The result of mean linear velocity.

\begin{tabular}{lccc}
\hline Number & $R_{e}$ & Mean linear velocity & Power correction factor \\
\hline 1 & 145 & 0.0816 & 1.067 \\
2 & 226 & 0.123 & 1.105 \\
3 & 459 & 0.231 & 1.196 \\
4 & 1168 & 0.569 & 1.235 \\
5 & 1853 & 0.863 & 1.290 \\
\hline
\end{tabular}

TABLE 4: The relationship of time difference and $R_{e}$.

\begin{tabular}{lcc}
\hline Number & $R_{e}$ & $\Delta T_{\exp }$ \\
\hline 1 & 145 & 90.5 \\
2 & 226 & 202.2 \\
3 & 459 & 346.8 \\
4 & 1168 & 746.4 \\
5 & 1853 & 1119.2 \\
\hline
\end{tabular}

increases. On the basis of Table 3 and using the logarithm of fitting method in the Matlab software, we can fit the power factor and Reynolds number as follows:

$$
K=0.08444 \log \left(R_{e}\right)+0.6532 .
$$

The curve that indicates the relationship between the power factor and Reynolds number is drawn in Figure 12.

4.3. The Relationship of Time Difference and $R_{e}$. During the test, $\Delta T_{\exp }$ represents the time difference of downstream and upstream, the related experimental results are shown in Table 4.

Based on Table 4, draw the diagram of relationships among simulated time differences, testing time differences, and Reynolds number in Figure 13.

The simulation and test outputs have the same trend with Reynolds number, but there are some offsets in Figure 13; the related seasons can be listed as follows.

Firstly, when installing two transducers along $\mathrm{AB}$, some installation errors always exist.

Secondly, when building the finite element model of flow field, the meshing type and the size of grids will affect the accuracy and then generate the errors.

Thirdly, while using Fluent to simulate and calculate, the setting of related parameters in the laminar flow model will influence the accuracy of outputs.

It is effective to converge the tested and simulated results by improving the accuracy of meshing, setting the reasonable parameters and installation accuracy. 


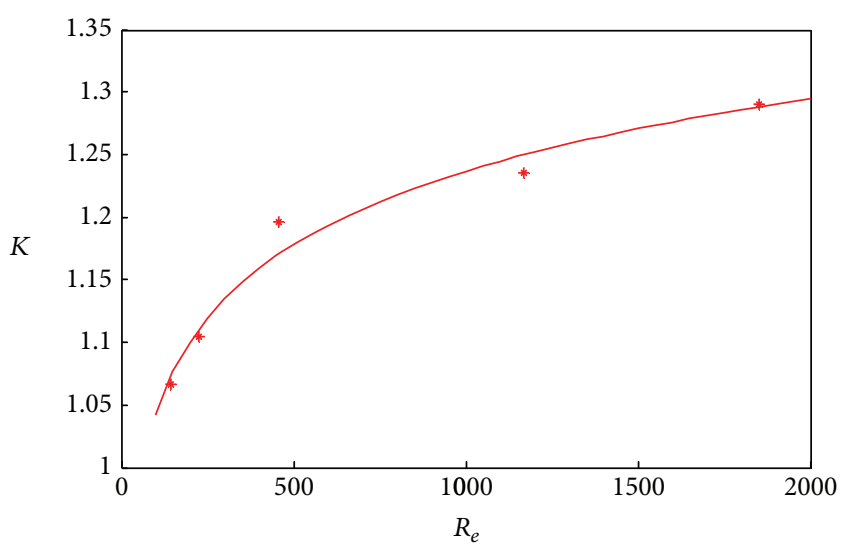

FIGURE 12: The relationship of power factor $K$ and $R_{e}$.

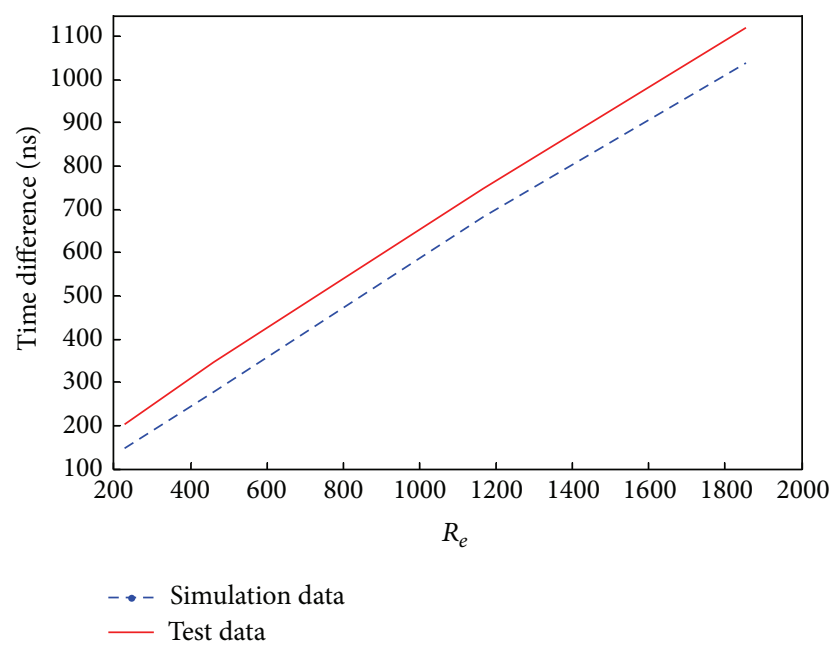

FIGURE 13: The relationship curve of time difference and $R_{e}$.

\section{Conclusion}

In this paper, we analyze the flow field of ultrasonic mono flowmeter with small diameter and low flow and discuss the influences on the flow field and power factor of exact pipe structure and the variation using different Reynolds number. The main conclusions are as follows.

(1) The installation point of ultrasonic transducer and temperature/pressure sensor will disturb the laminar flow field, the velocity will not be standard parabolic distribution any longer, and the reflux is generated at the transducer; the length of reflux has the same trend with Reynolds number.

(2) Near the transducer, the reflux decreases the linear average velocity and makes the measurement of flow lower; the errors will be negative.

(3) The expression of power correction factor by simulated data is fit.

(4) Through the test, the effectiveness of simulation is tested. Numerical simulation method can be a good reaction to flow state of flow field; it may be an important way to design and develop ultrasonic flowmeter.
In further work, we will take into account the main reason which causes the error between the test data and the simulation data and fit the power correction factor more accurately so that the proposed method is a more effective tool.

\section{Conflict of Interests}

The authors declare that there is no conflict of interests regarding the publication of this paper.

\section{Acknowledgment}

This work was supported by the Fundamental Research Funds for the Central Universities (no. CDJZR10170007).

\section{References}

[1] P.-Y. Zhang, D.-D. Zheng, T.-S. Xu, L.-X. Zhang, and H.-M. Hu, "Study on the influence of ultrasonic probes on flow field and measurement performance of ultrasonic flowmeter," Journal of Experiments in Fluid Mechanics, vol. 25, no. 3, pp. 60-65, 2011.

[2] L. C. Lynnworth, "Ultrasonic flow meters", Physical Acoustics: Principles and Methods, vol. 14, pp. 407-525, 1979.

[3] D. W. Spitzer, Flow Measurement: Practical Guides for Measurement and Control, Instrument society of Amer, 1993.

[4] D. Z. Huo, "Large flow-rate measurement and multichords ultrasonic flowmeter," in Proceedings of the Flow-Rate Measurement Conference, pp. 158-166, Zhengzhou, China, 2006.

[5] J. G. Drenthen and G. De Boer, "The manufacturing of ultrasonic gas flow meters," Flow Measurement and Instrumentation, vol. 12, no. 2, pp. 89-99, 2001.

[6] L. Zhang, T. Meng, C. Wang, H. M. Hu, and C. L. Qin, "Probe installation effects on the accuracy of feed thru ultrasonic flowmeters," Chinese Journal of Scientific Instrument, vol. 33, no. 10, pp. 2307-2314, 2012.

[7] C. Ruppel and F. Peters, "Effects of upstream installations on the reading of an ultrasonic flowmeter," Flow Measurement and Instrumentation, vol. 15, no. 3, pp. 167-177, 2004.

[8] M. L. Sanderson and H. Yeung, "Guidelines for the use of ultrasonic non-invasive metering techniques," Flow Measurement and Instrumentation, vol. 13, no. 4, pp. 125-142, 2002.

[9] Y. Hu, T. Zhang, and D. D. Zheng, "Estimation on influence of probe protrusion length of ultrasonic flowmeter on measurement," Journal of Tianjin University, vol. 46, no. 9, pp. 776-783, 2013.

[10] The American Society of Mechanical Engineer, ASME PTC182011 Hydraulic Turbines and Pump Turbines Performance Test Codes, The American Society of Mechanical Engineer, 2011.

[11] R. Raišutis, "Investigation of the flow velocity profile in a metering section of an invasive ultrasonic flowmeter," Flow Measurement and Instrumentation, vol. 17, no. 4, pp. 201-206, 2006.

[12] D. Zheng, P. Zhang, and T. Xu, "Effect of installation position of ultrasonic flowmeter probe on flow measurement," Journal of Mechanical Engineering, vol. 47, no. 12, pp. 13-18, 2011.

[13] L. Y. Zhong and L. Chang, "Modeling and simulating of multi path ultrasonic gas flowmeters," Journal of Huazhong University of Science and Technology, vol. 34, no. 4, 2006. 
[14] S. He, L. Peng, and H. Nakazato, "Computational fluid dynamics based sound path optimization for ultrasonic flow meter," Chinese Journal of Scientific Instrument, vol. 30, no. 4, pp. 852856, 2009.

[15] X. Wang and Z. Tang, "Model simulation and error quantitative analysis of pipeline of ultrasonic gas flowmeter," Chinese Journal of Scientific Instrument, vol. 30, no. 12, pp. 2612-2618, 2009.

[16] L. S. Bao, J. D. Liang, and J. Lu, "Mathematic model of multipath ultrasonic gas flowmeter," Technical Acoustics, vol. 29, no. 4, pp. 392-395, 2010.

[17] Y. Li and C. Li, "Modeling and simulating of multi-path ultrasonic gas flowmeters," Journal of Huazhong University of Science and Technology, vol. 34, no. 4, pp. 852-856, 2006.

[18] J. T. Liang, J. Lu, and S. M. Zhu, "Principles and calibrations of the multipath ultrasonic gas flowmeter," Chinese Journal of Scientific Instrument, vol. 22, no. 3, 2001.

[19] Y. L. Chen, C. M. Liu, C. Y. Chiang, S. M. Yuan, and J. H. Wang, "Building Communication Software: a project-based approach for teaching $\mathrm{C}^{++}$object-oriented Programming," International Journal of Innovative Computing, Information and Control, vol. 9, no. 8, pp. 3415-3436, 2013.

[20] K. Shirai, Y. Amano, and S. Omatu, "Process throughput analysis for manufacturing process under incomplete information based on physical approach," International Journal of Innovative Computing, Information and Control, vol. 9, no. 11, pp. 44314445, 2013. 


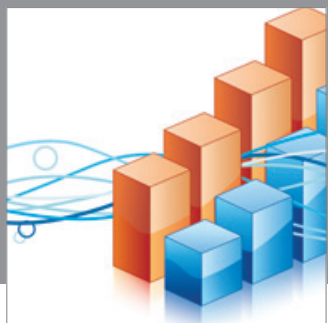

Advances in

Operations Research

mansans

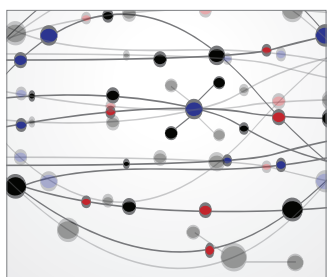

The Scientific World Journal
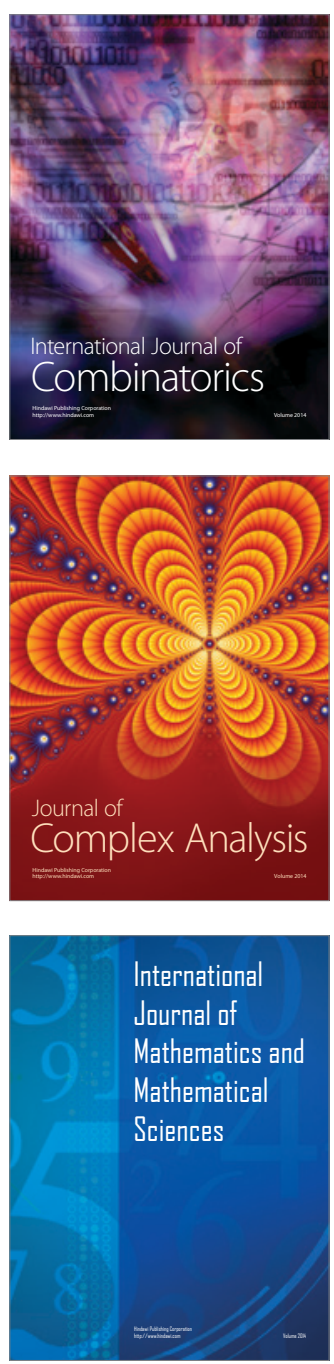
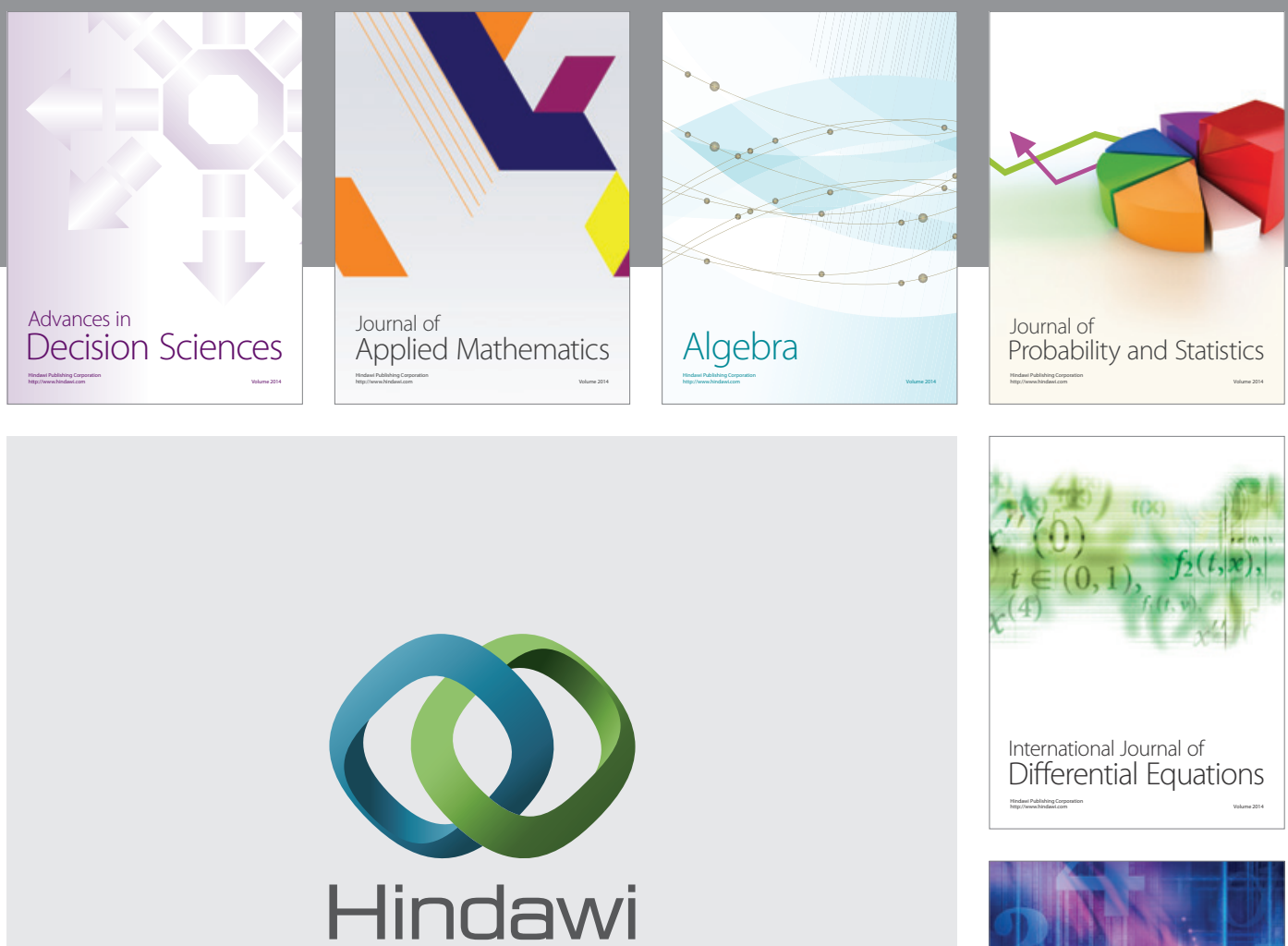

Submit your manuscripts at http://www.hindawi.com
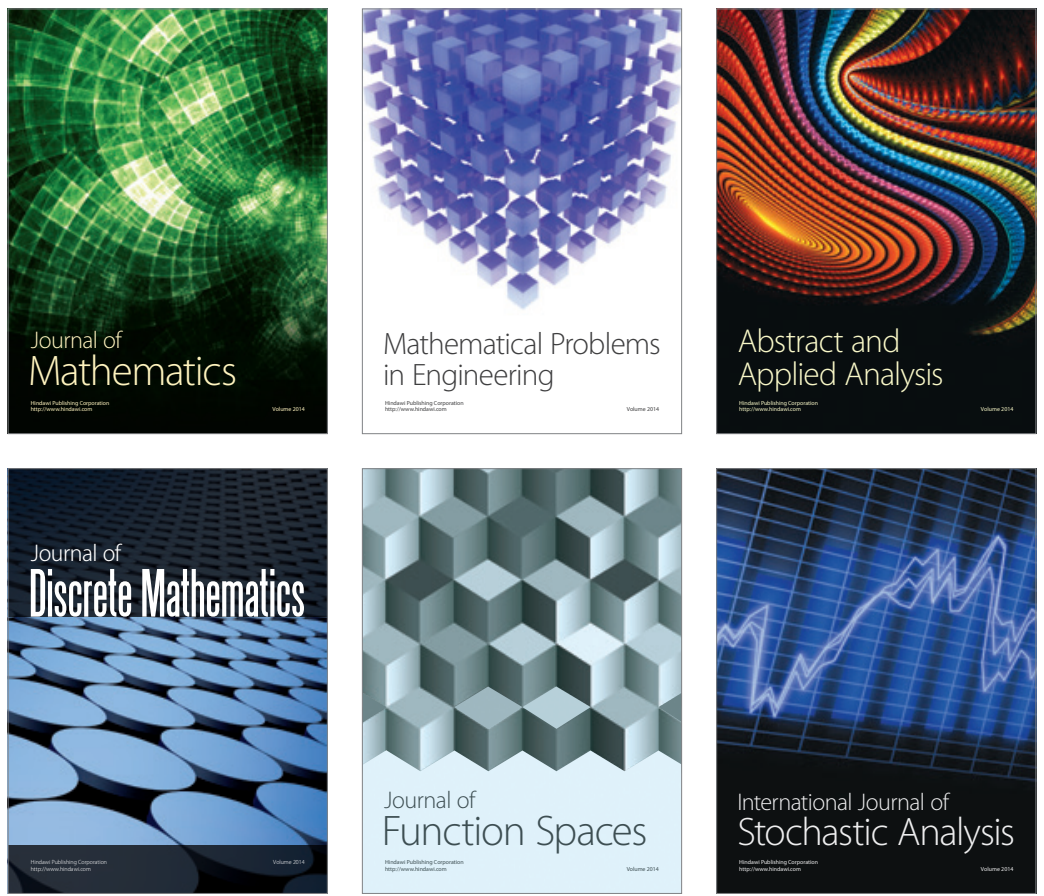

Journal of

Function Spaces

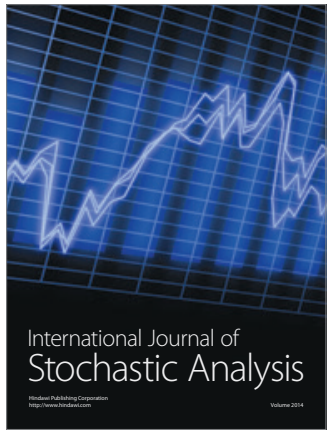

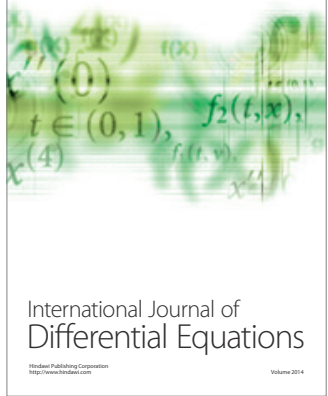
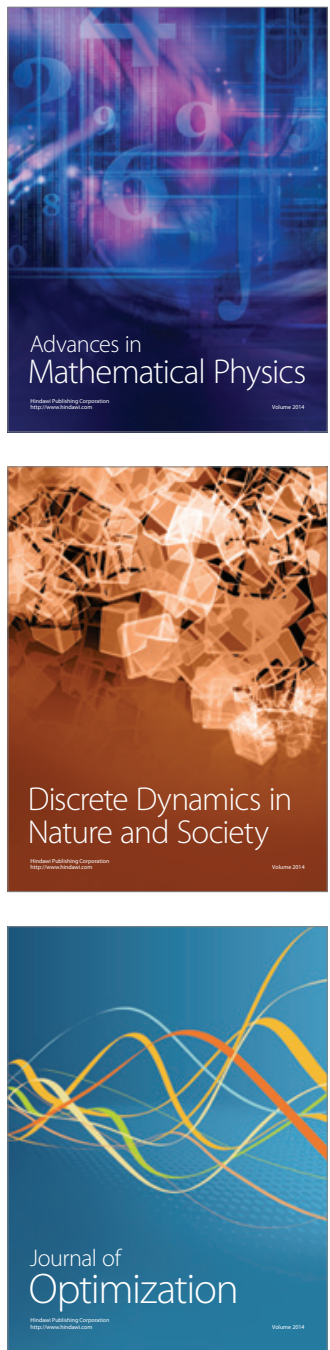